\title{
Magnetic vortex core reversal by excitation of spin waves
}

\author{
Matthias Kammerer', Markus Weigand1', Michael Curcic ${ }^{1}$, Matthias Noske1, Markus Sproll', Arne Vansteenkiste², \\ Bartel Van Waeyenberge ${ }^{2}$, Hermann Stoll', Georg Woltersdorf ${ }^{3}$, Christian H. Back ${ }^{3} \&$ Gisela Schuetz
}

Micron-sized magnetic platelets in the flux-closed vortex state are characterized by an in-plane curling magnetization and a nanometer-sized perpendicularly magnetized vortex core. Having the simplest non-trivial configuration, these objects are of general interest to micromagnetics and may offer new routes for spintronics applications. Essential progress in the understanding of nonlinear vortex dynamics was achieved when low-field core toggling by excitation of the gyrotropic eigenmode at sub-GHz frequencies was established. At frequencies more than an order of magnitude higher vortex state structures possess spin wave eigenmodes arising from the magneto-static interaction. Here we demonstrate experimentally that the unidirectional vortex core reversal process also occurs when such azimuthal modes are excited. These results are confirmed by micromagnetic simulations, which clearly show the selection rules for this novel reversal mechanism. Our analysis reveals that for spin-wave excitation the concept of a critical velocity as the switching condition has to be modified.

\footnotetext{
${ }^{1}$ Max-Planck-Institut für Metallforschung, Heisenbergstraße 3, 70569 Stuttgart, Germany. ${ }^{2}$ Department of Solid State Sciences, Ghent University, Krijgslaan 281 S1, 9000 Ghent, Belgium. ${ }^{3}$ Institut für Experimentelle und Angewandte Physik, Universität Regensburg, Universitätsstraße 31, 93053 Regensburg, Germany. Correspondence and requests for materials should be addressed to M.K. (email: kammerer@mf.mpg.de).
} 
$\mathrm{T}$ hin-film soft magnetic platelets of suited micron or submicron size are characterized by an in-plane closed flux magnetization, minimizing the dipolar energy. However, at the centre, the exchange energy forces the magnetization out-of-plane in a small area of only a few exchange lengths in diameter ${ }^{1,2}$ creating the vortex core with a distinct polarization $p$, either up $(p=+1)$ or down $(p=-1)$. The vortex introduces an important sub-GHz mode to the excitation spectrum of the magnetization ${ }^{3}$, which corresponds to the gyrotropic motion of the vortex.

Recently, dynamic vortex core reversal was discovered by application of pulsed magnetic fields $s^{4-6}$, alternating magnetic fields $\mathrm{s}^{7-10}$ or spin-polarized currents ${ }^{11,12}$. These discoveries did not only open up the possibility of using magnetic vortices as memory bits and for further spintronics applications ${ }^{13-15}$, but also initiated wide investigations of the physics behind this reversal mechanism.

The reversal process appeared to be universal and independent of the type of excitation: it proceeds through the creation and subsequent annihilation of a vortex-antivortex pair ${ }^{5,7,16}$. The source of this pair is the dynamical distortion of the otherwise stable vortex: it forms a 'dip'-an oppositely polarized region close to the moving vortex core ${ }^{17,18}$. Guslienko et al. ${ }^{16}$ explained this 'dip'formation by an effective field, the gyrofield, which is proportional to the vortex core velocity. This leads to the concept of a critical velocity for switching, which is about $320 \mathrm{~m} \mathrm{~s}^{-1}$ in Permalloy ${ }^{16}$.

Furthermore, the sense of rotation of a freely gyrating vortex is determined by the vortex core polarization ${ }^{3,19,20}$, a property which manifests itself in a high asymmetry of the gyrotropic resonance excited by rotating magnetic fields with different rotation senses ${ }^{9}$. This can be utilized to selectively reverse the core polarization, as shown experimentally by Curcic et al. ${ }^{8}$ Micromagnetic simulations from Kravchuk et al..$^{21,22}$ have indicated that rotating magnetic fields can even induce vortex core reversal at GHz frequencies as a result of the vortex spin wave interaction.

Here we present experiments which verify that polarization selective vortex core reversal can indeed be achieved by spin-wave excitation with in-plane rotating $\mathrm{GHz}$ magnetic fields. However, compared to the results of Kravchuk et al..$^{21}$ in which only one spin wave mode was considered, we will present selection rules for different modes. Furthermore, in contrast to the calculations given by Kravchuk et al..$^{22}$ in which the vortex core was artificially fixed during spin wave excitation, we will demonstrate by micromagnetic simulations that the core motion is essential and explains the differences observed in the vortex core switching triggered by distinct spin waves.

\section{Results}

The presence of the vortex core does not only introduce the gyrotropic mode, but also modifies the high-frequency spin wave normal mode spectrum ${ }^{23-26}$ by lifting the degeneracy of the azimuthal modes with opposite rotation sense ${ }^{27-32}$. The out-of-plane component of the magnetization $\Delta m_{z}$ of these azimuthal spin waves can be described in polar coordinates $(\rho, \varphi)$ as $^{24}$ :

$$
\Delta m_{z, n, m}(\rho, \phi, t)=r_{z, n}(\rho) e^{i m \phi} e^{-i \omega_{n, m, p^{t}}}
$$

These modes are characterized by a radial mode number $n$ (positive integer values starting with 1 ) denoting the number of nodes of the radial part within the disc and an azimuthal mode number $m$ (integer values), with $|2 m|$ being the number of nodes in the azimuthal part. The sign of $m$ denotes the rotation sense of the azimuthal wave (counterclockwise (CCW): $m$ positive, clockwise (CW): $m$ negative). The symmetry in the frequency splitting of the azimuthal mode can be written as $\omega_{n, m,-p}=\omega_{n,-m, p}$.

A general concept of resonant vortex core reversal by rotating magnetic fields is sketched in Figure 1. On the left-hand side, the

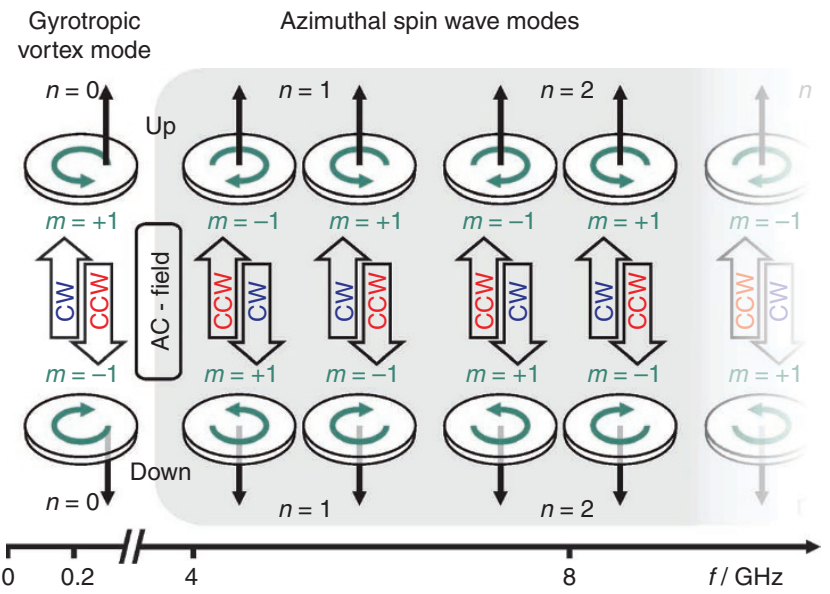

Figure 1 | Illustration of unidirectional vortex core reversal by external in-plane rotating magnetic fields. Switching only occurs if the senses of rotation (CW or CCW) of both the external field and the eigenmode (green arrows) are the same. At the left hand side, the sub-GHz frequency gyromode is illustrated. The right hand side shows the azimuthal spin wave modes at much higher $(\mathrm{GHz})$ frequencies, characterized by the radial mode number $n$ and the azimuthal mode number $(m= \pm 1)$, denoting the sense of rotation of the eigenmode. In vortex structures, the symmetry is broken by the out-of-plane component of the core, and thus, a frequency splitting is observed between $(m=-1)$ and $(m=+1)$ modes.

gyrotropic mode $(n=0, m \pm 1)$ is shown with a CCW sense of rotation for a core up state, and a CW sense of rotation for a core down state. The vortex core can only be reversed resonantly by applying an external rotating magnetic field if its frequency and sense of rotation correspond to the mode, as already shown experimentally by Curcic et al. ${ }^{8}$. On the right hand side of Figure 1, the frequency-split azimuthal modes are shown. For a vortex up, this means that the mode with a CW sense of rotation, denoted by $(n, m=-1)$, has a lower frequency and the mode with a CCW sense of rotation, denoted by $(n, m=+1)$, has a higher frequency. For a vortex core down, the sign of the azimuthal mode number $m$, indicating the sense of rotation, is reversed for the higher and lower frequency modes compared with vortex core up, as sketched at the bottom part of Figure 1 . These spin wave modes with $(n, m= \pm 1)$ can be selectively excited by the application of rotating in-plane magnetic fields with the corresponding frequency and rotation sense. Thus, at a fixed $\mathrm{GHz}$ frequency the excitation is polarization selective in the same way as for the sub-GHz gyrotropic mode: only rotating fields with one rotation sense will pump the system. This suggests that a similar polarization selective switching mechanism for the vortex core may exist when any of the azimuthal spin wave modes are excited, albeit at much higher frequencies.

Experimental results. We excited ferromagnetic Permalloy discs, $1.6 \mu \mathrm{m}$ in diameter, $50-\mathrm{nm}$ thick, in the vortex magnetic ground state with bursts of $\mathrm{GHz}$ rotating magnetic fields of variable frequency and amplitude. The fields were applied with either a CW or CCW rotation sense ${ }^{8}$. Magnetic scanning soft X-ray microscopy at the MAXYMUS endstation, Helmholtz Zentrum Berlin, BESSY II was used to determine the out-of-plane magnetization of the vortex core.

The experimental verification of the concept as described before is presented in Figure 2a. It shows the vortex core switching events from the initial vortex core up state to the down state as a function of excitation frequency, amplitude $B_{0}$ and sense of rotation of the external field. Here, the burst length was fixed at 


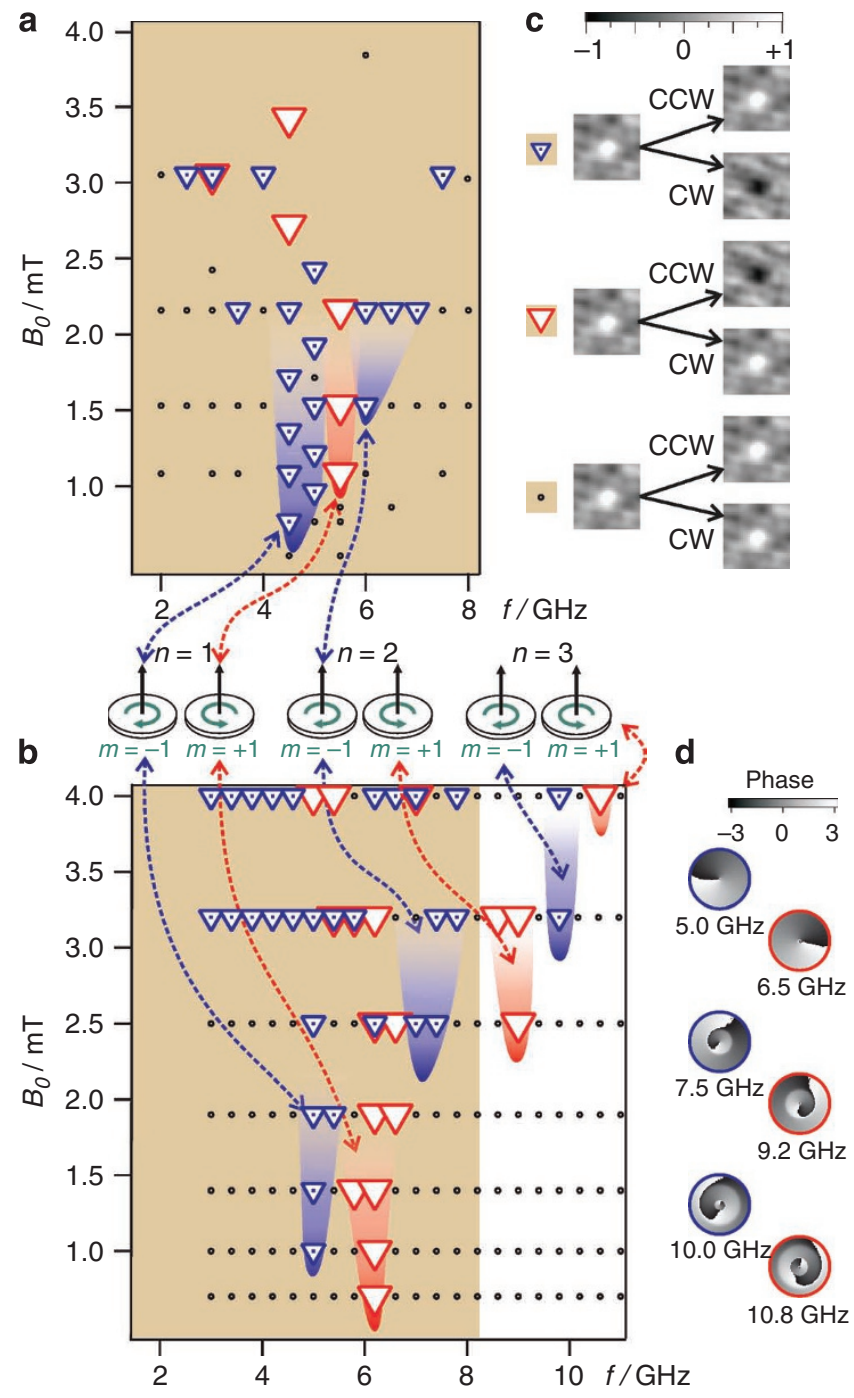

Figure $\mathbf{2}$ | Switching phase diagrams. The legend (c) shows microscopy images of X-ray transmission through the inner part $(150 \mathrm{~nm} \times 150 \mathrm{~nm})$ of the sample indicating the vortex core polarity (white: vortex up; black: vortex down) before and after a field burst. The phase diagrams show the points (excitation amplitude versus frequency) where vortex core reversal from up to down was observed in the experiments (a) and the simulations (b). Rotating in-plane magnetic field bursts with an amplitude $B_{0}$,

a frequency $f$ and a duration of 24 periods have been applied. As indicated in the legend of the top panel with the recorded X-ray images (c), the blue triangles with a dot in the middle indicate vortex core switching only after a $\mathrm{CW}$ rotating field burst, whereas red triangles indicate switching only after a CCW field burst. Black dots indicate no switching for either rotation sense. The minima in the switching threshold with differing sense of rotation correspond to the resonance frequencies of the excited azimuthal spin wave modes with the same sense of rotation as sketched in the middle of the figure. The modes are identified with the help of the phases derived from a local fast Fourier transform of the simulated out-of-plane magnetization of the sample with $1.62 \mu \mathrm{m}$ in diameter as shown in the inset to the right of the bottom panel (d).

24 periods of the excitation frequency. Three regions with a minimum in amplitude for vortex core reversal can be observed in the experimental data. Dependent on the sense of rotation of the external magnetic field they are marked with blue or red backgrounds. The regions for $\mathrm{CW}$ excitation at about 4.5 and $6.5 \mathrm{GHz}$, CCW excitation at about $5.5 \mathrm{GHz}$ can be assigned to the $(n=1$,

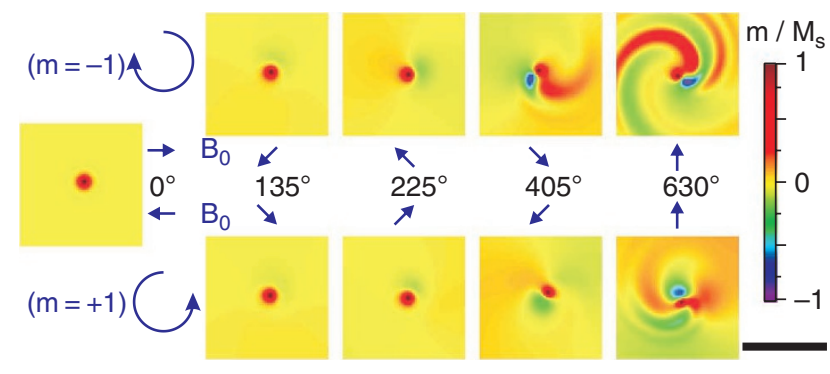

Figure 3 | Snapshots during spin-wave excitation before vortex core reversal. The frames show the time evolution of the out-of-plane magnetization for a vortex up during the application of in-plane rotating magnetic fields. Only the inner part of the sample is shown. The size of the black bar corresponds to a length of $200 \mathrm{~nm}$. The left frame corresponds to the relaxed ground state (phase angle $0^{\circ}$ ). The two rows oppose counter rotating modes at a frequency of $5.0 \mathrm{GHz}$ for the $(m=-1)$ mode and $6.2 \mathrm{GHz}$ for the $(m=+1)$ mode at the same azimuthal angle of the external field. The blue arrows in the middle indicate this angle for the corresponding frame.

$m=-1),(n=2, m=-1)$ and $(n=1, m=+1)$ spin wave modes, respectively (see Fig. 1 and the middle part of Fig. 2).

Micromagnetic simulations. To further investigate the core reversal at these different modes, we performed micromagnetic simulations $^{33}$ for the same sample size and experimental conditions. The results are shown in Figure $2 b$ and are in good agreement with our experimental results. The small differences between the experimental and simulated results can be attributed to the uncertainty in the thickness, saturation magnetization and damping coefficient of the material, as well as to possible structural imperfections in the samples. The phases of a local Fourier analysis ${ }^{23,25}$ of the dynamic magnetization let us clearly identify the azimuthal modes (see Fig. 2d). The simulations with an extended frequency range up to $10.5 \mathrm{GHz}$ reveal even more spin wave eigenmodes capable of vortex core reversal: the $(n=2, m=+1)$ mode at about $9 \mathrm{GHz}$ and the two $(n=3)$ spin wave modes at about 9.8 and $10.6 \mathrm{GHz}$.

Both the experiments and the micromagnetic simulations show that resonant switching of the vortex core from the down state back to the up state at the same frequency can be achieved only if the sense of rotation of the external field is reversed. This allows selective and unidirectional switching at each resonance frequency.

Detailed analysis of the micromagnetic simulations reveal that the excitation of all spin wave modes also leads to the creation of a 'dip' near the vortex core, resulting in the same core reversal mechanism as found for gyrotropic excitation ${ }^{5,7,17}$. It is based on the creation and annihilation of a vortex-antivortex pair. But the 'dip' creation itself differs dependent on which spin wave mode is excited. This 'dip' creation for a vortex up $(p=+1)$ is illustrated by snapshots of the simulations in Figure 3 for the modes $(n=1, m= \pm 1)$. In the first frame $\left(0^{\circ}\right)$, the unperturbed out-of-plane magnetization of the vortex can be seen. After a short time of excitation $\left(135^{\circ}\right.$ phase angle) the azimuthal spin wave with the same rotation sense as the external field develops. The interaction of the spin waves with the gyrotropic mode leads to a displacement of the vortex core towards the oppositely polarized region of the bipolar mode, resulting in a gyration with the same frequency as the external field $\left(225^{\circ}\right.$ phase angle). At $405^{\circ}$, a negatively polarized out-of-plane region can be observed next to the core, to the outside with respect to the centre. It is gyrating around the centre almost in phase with the vortex core. In the $(m=-1)$ case (upper panels), this region develops into a 'dip', which finally leads to core reversal. But surprisingly, for the mode $(m=+1)$ in the lower panels, there is an additional nega- 
a

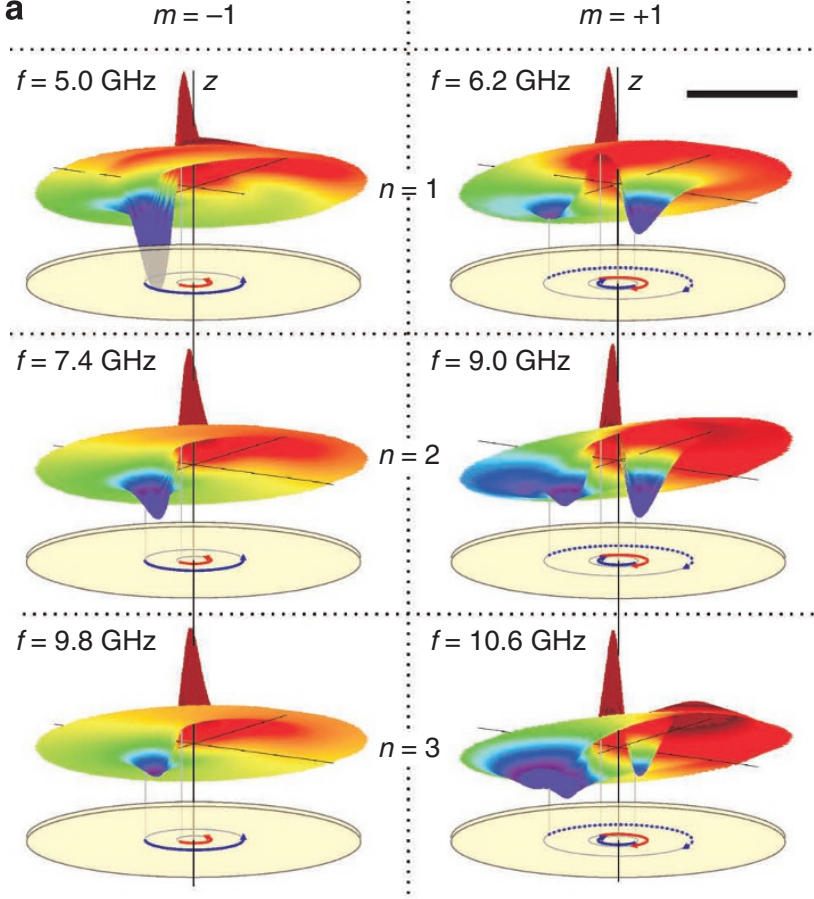

b

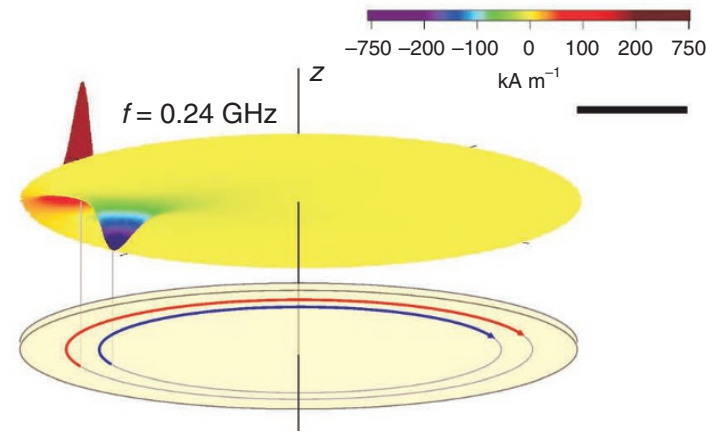

Figure 4 | Snapshots of the 'dip' structures. The out-of-plane magnetization of the central part extracted from the 1.6- $\mu \mathrm{m}$ disc before the core reversal. (a) A section of $300 \mathrm{~nm}$ in diameter showing the excited azimuthal spin wave modes as well as the 'dip'. The dynamics of modes with the same rotation sense are similar in the number of 'dips' and in their phase relation to the vortex core. This is indicated by the arrows below the snapshots. The difference between oppositely rotating modes are the result of the symmetry breaking due to the gyrofield. (b) For comparison the central part of the same structure with $500 \mathrm{~nm}$ in diameter is given, excited at the gyrotropic resonance. This results in a much larger trajectory of the core and the 'dip'. The black bars correspond to a lateral size of $100 \mathrm{~nm}$ in both cases $(\mathbf{a}, \mathbf{b})$

tively polarized region. This region is situated towards the structure centre where the azimuthal mode has a positive polarization and gyrates with a phase shift of about $180^{\circ}$ with respect to the vortex core. As can be seen from the last frame at $630^{\circ}$, it is this region which evolves into a fully out-of-plane 'dip', creating the vortexantivortex pair for switching. The typical magnetization configurations are shown in Figure 4, including the higher radial mode numbers. The trajectories for the vortex core, as well as for the 'dips', and their relative phases are illustrated by arrows.

\section{Discussion}

The gyrofield, as used by Guslienko et al. to describe the 'dip' formation in the gyrotropic mode ${ }^{19,16}$, can also be taken to explain the difference in 'dip' formation for the $(m=+1)$ and $(m=-1)$ modes, as is sketched in Figure 5. This effective field originates from the movement of the vortex and results in out-of-plane contributions to the left and right side of the core (with respect to the direction of motion), which are negative and positive, respectively (middle sketch in Fig. 5). This velocity dependent behaviour breaks the symmetry between counter rotating vortices. If we now combine the spin wave amplitude with the effect of the gyrofield, we can understand the 'dip' formation for any of the counter rotating modes (right sketch in Fig. 5). For a vortex up and the mode $(m=-1)$ with a CW vortex motion, the gyrofield acts constructively on the spin wave amplitude. For the $(m=+1)$ mode, it acts destructively, causing the double 'dip' configuration as seen in Figure 4. Alternatively, these 'dip' structures can be regarded as a hybridization between the gyrotropic mode and the azimuthal modes ${ }^{32}$. Kravchuk et al. ${ }^{22}$ also presented a 'dip' formation mechanism for spin-wave excitation under the condition of an artificially fixed vortex core. However, our results show that this condition can not be used to predict the reversal in free vortices, as in realistic structures. According to their analysis, the polarization direction of the 'dip' is given by the rotation sense of the external field only. This would mean that reversal of a given core polarization is only possible for one of the two azimuthal modes. For example, the reversal at the $(m=+1)$ as presented in Figure 2 can not be explained with this assumption, as the 'dip' polarization would be the same as the core polarization. The dynamic origin of the 'dip' structure clearly explains this contradiction. For a free vortex, the 'dip' formation seems to be dominated by the gyrofield.

Vortex core reversal by the low-frequency gyromode excitation results in a typical gyration radius of about $300 \mathrm{~nm}$ for this kind of sample. In contrast, the gyration radius of the vortex core at $\mathrm{GHz}$ frequencies is only in the order of $10 \mathrm{~nm}$ (see Fig. 4). But because of the high-frequency rotation, this small radius still results in a very high velocity of the vortex core. Figure 6 a shows the velocities of the vortex core directly before the reversal process as calculated from the simulations. It varies between about $100 \mathrm{~m} \mathrm{~s}^{-1}(m=-1)$ and up to more than $600 \mathrm{~m} \mathrm{~s}^{-1}(m=+1)$. This demonstrates that for $\mathrm{GHz}$ spin-wave excitation, no well-defined critical velocity is observed as it was calculated to be about $320 \mathrm{~m} \mathrm{~s}^{-1}$ for the gyromode of a Permalloy nanodot ${ }^{16}$.

The reason can be found in our explanation of the 'dip' formation as a combination of the gyrofield with the spin-wave amplitude. Although in first order, the gyrofield is proportional to the vortex velocity, the spin-wave amplitude is not, and thus the critical velocity before switching is lower or higher than the expected value, depending if their combination is constructive or destructive. Similar arguments were used to explain the changes in critical velocity when perpendicular bias fields are applied ${ }^{34}$. The large fluctuations in the velocities can be understood when the random contribution of short wavelength spin waves are also taken into account. Consequently, the velocity itself is not a switching criterion for the spin wave mode induced vortex core reversal.

Figure $6 \mathrm{~b}$ shows how long it takes for the vortex to switch after the burst is applied. It is no surprise that the larger the amplitude, the shorter the time for vortex core reversal. The minima in the excitation amplitude show a clear resonant behaviour. At high amplitudes the switching times become smaller than one period of the excitation and the frequency dependence is washed out, indicating the transition to non-resonant excitation ${ }^{5}$. However, the feature of unidirectionality gets lost in the intersection of two oppositely rotating modes if too short bursts are applied.

Because both the vortex and the created vortex-antivortex pair remain close to the centre of the structure during the excitation, the relaxation after the spin wave-induced reversal process is much faster than in the gyrotropic case, in which it has to spiral back from its orbit far away to the centre. In combination with the very small gyration radius, this fast relaxation is accelerating the whole reversal 


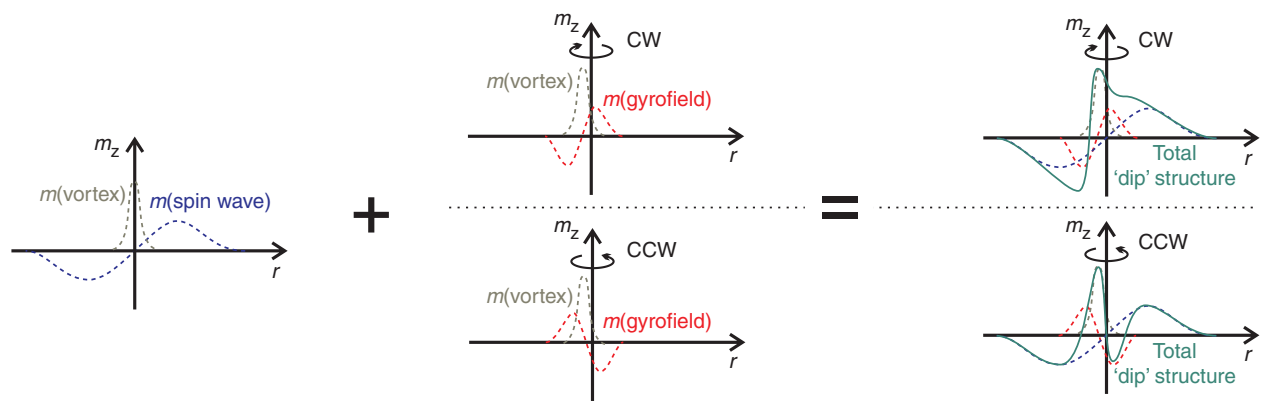

Figure $\mathbf{5}$ | Model for spin wave-induced vortex core reversal. This sketch shows the origin of the out-of-plane magnetization near the vortex core and illustrates the origin of the observed asymmetry in the 'dip' formation for CW versus CCW spin-wave excitation. To the left, the basic shape of the vortex core and the bipolar amplitude of the spin wave are shown. The magnetization change as a result of the gyrofield of the moving vortex core differs for CW and CCW rotation senses (middle sketches). The resulting structures (right sketches) agree qualitatively with results from the micromagnetic simulations as shown in Figures 3, 4, and 6.
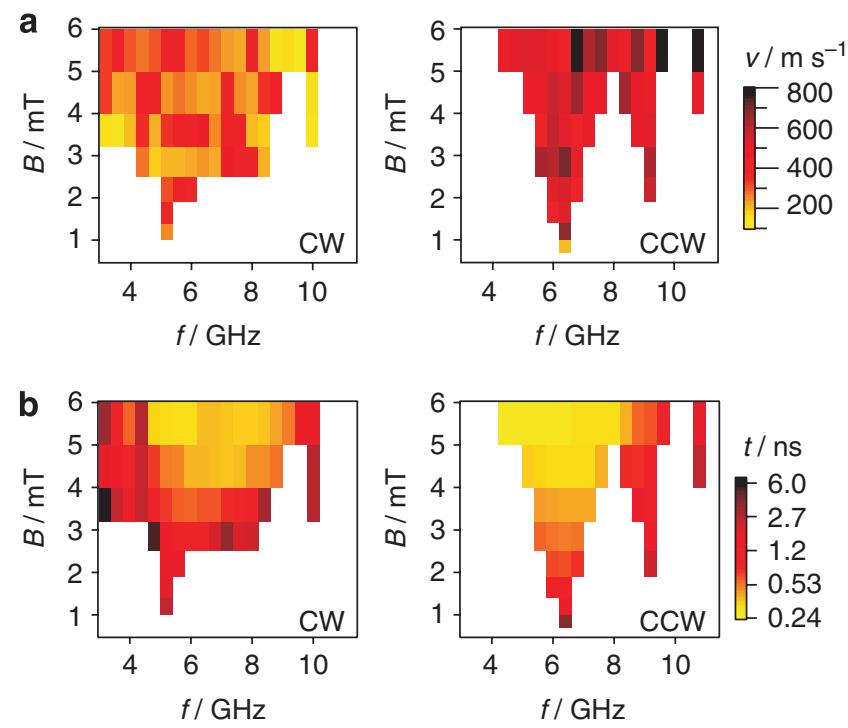

Figure 6 | Vortex core velocities and switching times. (a) Vortex core velocity just before switching. The gyrofield of the moving vortex is proportional to this velocity. Because of the important contribution of the spin wave background, this quantity is not a constant at $\mathrm{GHz}$ excitation and shows strong differences between $\mathrm{CW}$ and CCW excitation. (b) Excitation time until switching occurs in a logarithmic colour scale. At sufficiently high amplitudes, switching takes on the order of one period of the excitation frequency, resulting in a widening of the resonances and a dominance of the mode $(n=1, m=+1)$.

process by up to two orders of magnitude, while the total energy pumped into the system is roughly the same compared with reversal at the gyrotropic mode. This fact makes this new reversal process very beneficial for fast technological applications.

In conclusion, our experiments show vortex core reversal at $\mathrm{GHz}$ frequencies. By taking advantage of the vortex polarity-dependent frequency splitting of the azimuthal modes $(n, m= \pm 1)$, we present a concept for unidirectional reversal by exciting any of these modes up to $11 \mathrm{GHz}$. Although the dynamics depend significantly on the different combinations of core polarity and azimuthal mode number, they can be explained by the superposition of the gyrofield with the spin-wave amplitude. Consequently, immediately before the switching event, different vortex core velocities are observed, which excludes the velocity as a critical condition for core reversal at $\mathrm{GHz}$ excitation. This finding highlights the importance of spin wave-vortex interaction and boosts vortex core reversal to much higher frequencies, which may offer new routes for spintronics applications.

\section{Methods}

Experiments. Permalloy discs, $1.6 \mu \mathrm{m}$ in diameter and $50 \mathrm{~nm}$ thickness, are grown on top of $2.5-\mu \mathrm{m}$ wide and $170-\mathrm{nm}$ thick copper striplines patterned on a $100-\mathrm{nm}$ thick silicon nitride membrane. All structures are prepared by electron-beam lithography, thermal evaporation and lift-off processes. AC currents with a phase shift of $\pm 90^{\circ}$ flowing through orthogonal striplines are generating rotating in-plane magnetic fields ${ }^{8}$. The out-of-plane magnetization of the Permalloy disc was imaged by scanning transmission X-ray microscopy at the MAXYMUS end station at HZB BESSY II, Berlin, by taking advantage of the X-ray magnetic circular dichroism ${ }^{35}$. The instrument has a resolution of about $25 \mathrm{~nm}$, which is sufficient to resolve the vortex core and its polarization.

Simulations. Micromagnetic simulations were performed with the object oriented micromagnetic framework ${ }^{33}$. A disc, $1.62 \mu \mathrm{m}$ in diameter and $50-\mathrm{nm}$ thick, was simulated with a cell size of $3 \times 3 \times 50 \mathrm{~nm}^{3}$. Typical material parameters for Permalloy ${ }^{6,8}$ were used: $M_{\mathrm{s}}=750 \times 10^{3} \mathrm{~A} \mathrm{~m}^{-1}$, exchange constant $A=13 \times 10^{-12} \mathrm{~J} \mathrm{~m}^{-1}$, damping parameter $\alpha=0.006$, anisotropy constant $K_{1}=0$. For all simulations shown, the initial state was a vortex core up. The symmetry of the results were verified by also simulating with the opposite initial state, which is a vortex down. The sample was excited by homogeneous in-plane rotating magnetic field bursts with duration of 24 periods. The frequency of the bursts was scanned from 3 to $11 \mathrm{GHz}$ in $0.4 \mathrm{GHz}$ steps for both, CW and CCW sense of rotation.

\section{References}

1. Shinjo, T., Okuno, T., Hassdorf, R., Shigeto, K. \& Ono, T. Magnetic vortex core observation in circular dots of permalloy. Science 289, 930-932 (2000).

2. Wachowiak, A. et al. Direct observation of internal spin structure of magnetic vortex cores. Science 298, 577-580 (2002).

3. Huber, D. Equation of motion of a spin vortex in a two-dimensional planar magnet. J. Appl. Phys. 53, 1899-1900 (1982).

4. Xiao, Q., Rudge, J., Choi, B., Hong, Y. \& Donohoe, G. Dynamics of vortex core switching in ferromagnetic nanodisks. Appl. Phys. Lett. 89, 262507 (2006).

5. Hertel, R., Gliga, S., Fahnle, M. \& Schneider, C. Ultrafast nanomagnetic toggle switching of vortex cores. Phys. Rev. Lett. 98, 117201 (2007).

6. Weigand, M. et al. Vortex core switching by coherent excitation with single in-plane magnetic field pulses. Phys. Rev. Lett. 102, 077201 (2009).

7. Van Waeyenberge, B. et al. Magnetic vortex core reversal by excitation with short bursts of an alternating field. Nature 444, 461-464 (2006).

8. Curcic, M. et al. Polarization selective magnetic vortex dynamics and core reversal in rotating magnetic fields. Phys. Rev. Lett. 101, 197204 (2008).

9. Lee, K. \& Kim, S. Two circular-rotational eigenmodes and their giant resonance asymmetry in vortex gyrotropic motions in soft magnetic nanodots. Phys. Rev. B Condens. Matter Mater. Phys. 78, 014405 (2008).

10. De Loubens, G. et al. Bistability of vortex core dynamics in a single perpendicularly magnetized nanodisk. Phys. Rev. Lett. 102, 177602 (2009).

11. Yamada, K. et al. Electrical switching of the vortex core in a magnetic disk. Nat. Mater. 6, 269-273 (2007).

12. Yamada, K., Kasai, S., Nakatani, Y., Kobayashi, K. \& Ono, T. Switching magnetic vortex core by a single nanosecond current pulse. Appl. Phys. Lett. 93, 152502 (2008). 
13. Kim, S., Lee, K., Yu, Y. \& Choi, Y. Reliable low-power control of ultrafast vortex-core switching with the selectivity in an array of vortex states by in-plane circular-rotational magnetic fields and spin-polarized currents. Appl. Phys. Lett. 92, 022509 (2008).

14. Bohlens, S. et al. Current controlled random-access memory based on magnetic vortex handedness. Appl. Phys. Lett. 93, 142508 (2008).

15. Pigeau, B. et al. A frequency-controlled magnetic vortex memory. Appl. Phys Lett. 96, 132506 (2010).

16. Guslienko, K., Lee, K. \& Kim, S. Dynamic origin of vortex core switching in soft magnetic nanodots. Phys. Rev. Lett. 100, 027203 (2008).

17. Vansteenkiste, A. et al. X-ray imaging of the dynamic magnetic vortex core deformation. Nat. Phys. 5, 332-334 (2009).

18. Völkel, A. R., Wysin, G. M., Mertens, F. G., Bishop, A. R. \& Schnitzer, H. J. Collective-variable approach to the dynamics of nonlinear magnetic excitations with application to vortices. Phys. Rev. B 50, 12711-12720 (1994).

19. Thiele, A. A. Steady-state motion of magnetic domains. Phys. Rev. Lett. 30, 230-233 (1973).

20. Choe, S. B. et al. Vortex core-driven magnetization dynamics. Science 304, 420-422 (2004).

21. Kravchuk, V., Sheka, D., Gaididei, Y. \& Mertens, F. Controlled vortex core switching in a magnetic nanodisk by a rotating field. J. App. Phys. 102, 043908 (2007).

22. Kravchuk, V., Gaididei, Y. \& Sheka, D. Nucleation of a vortex-antivortex pair in the presence of an immobile magnetic vortex. Phys. Rev. B Condens. Matter Mater. Phys. 80, 100405 (2009).

23. Park, J., Eames, P., Engebretson, D., Berezovsky, J. \& Crowell, P. Imaging of spin dynamics in closure domain and vortex structures. Phys. Rev. B Condens. Matter Mater. Phys. 67, 020403 (2003).

24. Buess, M. et al. Excitations with negative dispersion in a spin vortex. Phys. Rev. B 71, 104415 (2005)

25. Buess, M. et al. Fourier transform imaging of spin vortex eigenmodes. Phys. Rev. Lett. 93, 077207 (2004).

26. Neudecker, I. et al. Modal spectrum of permalloy disks excited by in-plane magnetic fields. Phys. Rev. B 73, 134426 (2006).

27. Zaspel, C., Ivanov, B. J. \& Crowell, P. Excitations in vortex-state permalloy dots. Phys. Rev. B Condens. Matter Mater. Phys. 72, 024427 (2005).

28. Park, J. \& Crowell, P. Interactions of spin waves with a magnetic vortex. Phys. Rev. Lett. 95, 167201 (2005).

29. Zhu, X., Liu, Z., Metlushko, V., Grutter, P. \& Freeman, M. Broadband spin dynamics of the magnetic vortex state: Effect of the pulsed field direction. Phys. Rev. B Condens. Matter Mater. Phys. 71, 180408 (2005).

30. Hoffmann, F. et al. Mode degeneracy due to vortex core removal in magnetic disks. Phys. Rev. B Condens. Matter Mater. Phys. 76, 014416 (2007).
31. Guslienko, K. Y., Slavin, A. N., Tiberkevich, V. \& Kim, S.- K. Dynamic origin of azimuthal modes splitting in vortex-state magnetic dots. Phys. Rev. Lett. 101, 247203 (2008).

32. Guslienko, K., Aranda, G. \& Gonzalez, J. Topological gauge field in nanomagnets: Spin-wave excitations over a slowly moving magnetization background. Phys. Rev. B Condens. Matter Mater. Phys. 81, 014414 (2010).

33. Donahue, M. \& Porter, D. Oommf User's Guide, Version 1.0. Interagency Report NISTIR 6376.National Institute of Standards and Technology, (1999).

34. Yoo, M.- W., Lee, K.- S., Jeong, D.- E. \& Kim, S.- K. Origin, criterion, and mechanism of vortex-core reversals in soft magnetic nanodisks under perpendicular bias fields. Phys. Rev. B 82, 174437 (2010).

35. Schutz, G. et al. Absorption of circularly polarized x-rays in iron. Phys. Rev. Lett. 58, 737-740 (1987).

\section{Acknowledgments}

We thank Manfred Fähnle, MPI Stuttgart, Franz Mertens, Bayreuth University, Yuri Gaididei and Volodymyr Kravchuk, Bogolyubov Institute for Theoretical Physics, Kiev, and Denis Sheka, Kiev University, for fruitful discussions. We also thank all people involved in the construction and operation of the MAXYMUS scanning X-ray microscope at HZB, BESSY II in Berlin, in particular, Brigitte Baretzky, Michael Bechtel, Rolf Follath, Corinne Grévent and Eberhard Goering. Cooperation with Rolf Heidemann, Ulm University, in the stripline design for microwave application is gratefully acknowledged.

\section{Author contributions}

M.K., H.S., B.V.W. and G.S. planned the project; G.W. and C.H.B. prepared the samples; M.K., M.N., M.S., M.W. and M.C. carried out the experiments; micromagnetic simulations was done by M.K.; M.K., M.C., A.V., B.V.W. and H.S. analysed data; M.K., M.N., M.S., M.C., M.W., A.V., B.V.W., H.S., G.W., C.H.B. and G.S. wrote the paper.

\section{Additional information}

Competing financial interests: The authors declare no competing interests.

Reprints and permission information is available online at http://npg.nature.com/ reprintsandpermissions/

How to cite this article: Kammerer, M. et al. Magnetic vortex core reversal by excitation of spin waves. Nat. Commun. 2:279 doi: 10.1038/ncomms1277 (2011).

License: This work is licensed under a Creative Commons Attribution-NonCommercialShare Alike 3.0 Unported License. To view a copy of this license, visit http:// creativecommons.org/licenses/by-nc-sa/3.0/ 